\title{
Activity of Different Classes of Neurons of the Motor Cortex during Postural Corrections
}

\author{
Irina N. Beloozerova, ${ }^{2,3}$ Mikhail G. Sirota, ${ }^{2,3}$ Harvey A. Swadlow, ${ }^{2}$ Grigori N. Orlovsky, ${ }^{1}$ Lioudmila B. Popova, ${ }^{4}$ and \\ Tatiana G. Deliagina ${ }^{1}$ \\ ${ }^{1}$ Department of Neuroscience, Karolinska Institute, SE-171 77, Stockholm, Sweden, ${ }^{2}$ Department of Psychology, University of Connecticut, Storrs, \\ Connecticut 06269, ${ }^{3}$ Barrow Neurological Institute, Phoenix, Arizona 85013, and ${ }^{4}$ A. N. Belozersky Institute of Physico-Chemical Biology, Moscow State \\ University, Moscow 119 899, Russia
}

The dorsal side-up body orientation in quadrupeds is maintained by a postural system that is driven by sensory feedback signals. The spinal cord, brainstem, and cerebellum play essential roles in postural control, whereas the role of the forebrain is unclear. In the present study we investigated whether the motor cortex is involved in maintenance of the dorsal side-up body orientation. We recorded activity of neurons in the motor cortex in awake rabbits while animals maintained balance on a platform periodically tilting in the frontal plane. The tilts evoked postural corrections, i.e., extension of the limbs on the side moving down and flexion on the opposite side. Because of these limb movements, rabbits maintained body orientation close to the dorsal side up. Four classes of efferent neurons were studied: descending corticofugal neurons of layer V (CF5s), those of layer VI (CF6s), corticocortical neurons with ipsilateral projection (CCIs), and those with contralateral projection (CCCs). One class of inhibitory interneurons [suspected inhibitory neurons (SINs)] was also investigated. CF5 neurons and SINs were strongly active during postural corrections. In most of these neurons, a clear-cut modulation of discharge in the rhythm of tilting was observed. This finding suggests that the motor cortex is involved in postural control. In contrast to CF5 neurons, other classes of efferent neurons (CCI, CCC, CF6) were much less active during postural corrections. This suggests that corticocortical interactions, both within a hemisphere (mediated by CCIs) and between hemispheres (mediated by CCCs), as well as corticothalamic interactions via CF6 neurons are not essential for motor coordination during postural corrections.

Key words: posture; motor cortex; efferent neurons; interneurons; sensory motor integration; rabbit

\section{Introduction}

The dorsal side-up body orientation in quadrupeds is maintained because of the activity of a postural system. This closed-loop system operates on the basis of sensory feedback signals and generates a corrective motor response with any deviation of the body from the normal orientation (for review, see Horak and Macpherson, 1995; Macpherson et al., 1997; Massion, 1998).

Lesion and stimulation experiments indicate that basic nervous mechanisms for postural control reside in the brainstem, cerebellum, and spinal cord. First, it was shown that chronic decerebrate animals are able to maintain the dorsal side-up posture (Magnus, 1924; Bard and Macht, 1958). Second, brain stem stimulation affects the tone of extensor muscles (Mori, 1987). Third, after inactivation of the motor cortex, that is, of the main motor output of the forebrain, the animals are able to maintain equilibrium when standing or walking on a flat terrain (Cham-

\footnotetext{
Received March 19, 2003; revised June 13, 2003; accepted June 16, 2003.

This work was supported by the Swedish Medical Research Council (Grant 11554), the Royal Swedish Academy of Sciences, the Gösta Fraenckels Foundation, the National Science Foundation (Grant IBN-0077694), and National Institutes of Health (NIH) Grants F32 NS-10314, NIH R01 NS-39340, and MH-64024. We are grateful to Dr. Zelenin for valuable comments on this manuscript.

Correspondence should be addressed to Dr. T. G. Deliagina, The Nobel Institute for Neurophysiology, Department of Neuroscience, Karolinska Institute, SE-17177, Stockholm, Sweden. E-mail: tatiana.deliagina@neuro.ki.se. Copyright $\odot 2003$ Society for Neuroscience $\quad$ 0270-6474/03/237844-10\$15.00/0
}

bers and Lin, 1957; Adkins et al., 1971; Dubrovsky et al., 1974; Beloozerova and Sirota, 1988, 1993).

Thus, the integrity of the motor cortex is not necessary for the feedback mode of operation of the postural control system. It remained unclear, however, whether the motor cortex does contribute to postural control when it is intact (Horak and Macpherson, 1995).

Recording of neuronal activity during performance of a postural task is a different approach to clarification of the role of a motor center in postural control. That approach was applied to some invertebrate (Hensler, 1988; Deliagina et al., 1998, 1999) and lower vertebrate animals (Deliagina et al., 2000c; Zelenin et al., 2000) but not to mammalian species.

In the present study we address the question of whether the motor cortex is involved in the control of posture in the feedback mode by examining the activity of cortical neurons during postural corrections in the rabbit that maintained balance on a tilting platform. A choice of the experimental animal and postural task was determined by the following factors. (1) Rabbits could be engaged in this postural task without training and displayed a robust pattern of motor and muscle responses (Deliagina et al. 2000a). (2) The same animals were used for the study of cortical activity during locomotion in concurrent experiments (Beloozerova et al., 2003). This presented us with an opportunity to compare cortical involvement in the two different motor tasks. 
Five classes of neurons from the forelimb representation in the motor cortex were studied (see Fig. 1A): (1) descending corticofugal neurons of layer $\mathrm{V}$ (CF5) that are known to project to subcortical motor nuclei and the spinal cord (Kuypers, 1981; Wiesendanger, 1981; Jones, 1984); (2) corticofugal neurons of layer VI (CF6) projecting to the thalamus; (3) callosal efferent neurons (CCC) projecting to the contralateral motor or primary somatosensory cortex; (4) corticocortical efferent neurons (CCI) projecting to the ipsilateral primary somatosensory cortex; (5) putative GABAergic fast-spike inhibitory interneurons [suspected inhibitory neurons (SINs) (Swadlow, 1988, 1994)]. Examining such a wide spectrum of neuron classes allowed us to estimate a possible role of different cortical mechanisms in the generation of postural corrections.

A brief account of part of this study has been published previously in abstract form (Deliagina et al., 2000b).

\section{Materials and Methods}

Experiments were performed on adult, fully awake Dutch-belted rabbits. Some of the methods have been described previously (Swadlow, 1989, 1990; Beloozerova and Sirota, 1993; Beloozerova et al., 2003) and are reported briefly here. All experiments were conducted with the approval of the University of Connecticut Animal Care and Use Committee and the local ethical committee (Norra Djurförsöksetiska Nämnden) in Stockholm.

Surgery. Surgery was performed under pentobarbital sodium anesthesia (initial dose $25-35 \mathrm{mg} / \mathrm{kg}$ ) using aseptic procedures. After removal of skin and fascia above the dorsal surface of the skull, a metal ring was attached to the skull by means of screws and cement. The ring served as a base for a micromanipulator for an extracellular microelectrode, a preamplifier, contacts for EMG wires, and contacts for stimulating electrodes. The ring was also used for temporary fixation of the head during identification of neurons. Two electrodes for EMG recording (flexible 0.2 $\mathrm{mm}$ Teflon-insulated wires) separated by $10 \mathrm{~mm}$ were implanted into muscle $(\mathrm{m})$ triceps (elbow extensor) of the left and right forelimb. The wires were positioned under the skin and soldered to a connector on the ring base.

A portion of the skull over the approximate locations of the left motor and the primary somatosensory limb representations was mechanically thinned to $\sim 0.3 \mathrm{~mm}$. This allowed an easy removal of the remaining thinned bone over an area of 100-200 $\mu \mathrm{m}$ in diameter before a recording or a stimulation electrode was inserted into the cortex in an awake animal. Implantation of all stimulating electrodes (platinum-iridium wires, $125 \mu \mathrm{m}$ in diameter) was performed several days after the initial surgery, following a careful mapping of the target areas using multiple-unit recording procedures in an awake animal (Swadlow, 1989; Beloozerova et al., 2003). Two electrodes were implanted $1 \mathrm{~mm}$ apart in the forelimb representation of primary somatosensory cortex (S1) in the left hemisphere, as well as in the forelimb representation of motor cortex and S1 in the right hemisphere. Two or three electrodes were implanted within the forelimb representation of the left ventrolateral thalamus. The stimulated sites are schematically shown in Figure $1 A$ (Stim 1-Stim 4).

Identification of the region of motor cortex. Motor cortex was identified in awake animals by first localizing the forepaw representation of the primary somatosensory cortex using multiple-unit receptive field mapping procedures (Gould, 1986; Swadlow, 1990). During mapping, microelectrode penetrations were made $300-1000 \mu \mathrm{m}$ apart with a tungsten varnish insulated electrode [50 $\mu \mathrm{m}$ outer diameter (OD)]. Somatosensory receptive fields and effects of microstimulation were examined in layer IV of the primary somatosensory cortex and in layer $\mathrm{V}$ of the motor cortex. Differentiation of motor from somatosensory cortex was based on (1) more rostral location (Fig. $1 B, C$ ), (2) a gross prevalence of deep (muscle and joints) over superficial (skin) receptive fields, (3) much larger size of receptive fields, (4) lower current intensities that were required to evoke limb movements, and (5) a poorly defined granular layer IV and a larger size of pyramids in layer $\mathrm{V}$. The forelimb area of the motor cortex (Fig. 1C, 1) was found on the rostral-medial border of the fore- limb representation of the primary somatosensory cortex (Fig. 1C, 2a). Here, cells responded only to deep manipulations of the limb (joints or muscles or both), microstimulation (trains of five 50-100 $\mu \mathrm{A}$ cathodal pulses at $100 \mathrm{~Hz}$ ) resulted in primarily flexion movements of the limb, and the granular layer was virtually absent. In all animals tested, motor limb representation was separated from the sensory limb representation by a stripe of nonresponsive tissue.

Cell recording and identification. At the beginning of an experiment, the rabbit was put on a foam pad and its head was fixed in a headrestraining device by the base that was attached to the skull during surgery. Methods ensuring the humane treatment of subjects during immobilization of the head have been described (Swadlow, 1988, 1994). All procedures related to microelectrode recordings were described in detail by Beloozerova et al. (2003). In brief, a small entrance $(100-200 \mu \mathrm{m}$ in diameter) for insertion of the microelectrode was drilled into the skull above the motor cortex in the left hemisphere. The microelectrodes were fabricated from platinum-tungsten wire insulated with quartz glass (40 $\mu \mathrm{m}$ OD) and had impedance of $2-4 \mathrm{M} \Omega$ at $1000 \mathrm{~Hz}$. The preamplified signal from the microelectrode as well as the signals from the EMG electrodes were led to recording devices by a flexible cable. They were amplified by conventional AC amplifiers. The signals were then passed through filters $(300-10,000 \mathrm{~Hz}$ bandpass for spikes and $300-1000 \mathrm{~Hz}$ bandpass for EMG), digitized with a sampling frequency of $30 \mathrm{kHz}$, and recorded to the disk of an IBM AT compatible computer using data acquisition software (Digidata 1200/Axoscope, Axon Instruments, Foster City, CA). In addition, spike signals were led to an oscilloscope for display and analysis of synaptic or antidromic responses. To aid in the identification of single neurons, the waveform analysis was used to discriminate and identify the spikes of the neuron using the Spike2 system waveformmatching algorithm (Cambridge Electronic Design, Cambridge, UK).

All neurons were tested for antidromic activation using $0.2 \mathrm{msec}$ rectangular pulses of graded intensity in the range of $0.1-2 \mathrm{~mA}$. The principal criterion for the identification of antidromic activation was the test for collision of spikes (Bishop et al., 1962; Fuller and Schlag, 1976). Silent neurons that could not be tested for collision were considered to be antidromically activated if they satisfied two ancillary criteria: (1) a refractory period of $<2.0 \mathrm{msec}$ and (2) latency variability to a test stimulus of either $<0.1 \mathrm{msec}$ or $<1 \%$ of the antidromic latency, whichever is greater when the test stimulus followed a suprathreshold conditioning stimulus at an interval of $10 \mathrm{msec}$ (Swadlow, 1998). Five classes of neurons were identified using the criteria described in detail by Swadlow (1988, 1994).

Descending corticofugal neurons of layer V were identified by their antidromic response to stimulation of the ventrolateral thalamus. It can be assumed that CF5 neurons identified in this study also projected to other subcortical motor nuclei and spinal cord, because anatomical studies show that (1) efferent neurons of layer $\mathrm{V}$ never terminate only in thalamus, but in addition also project elsewhere, and (2) the cell bodies of corticofugal neurons that project to subcortical motor nuclei and spinal cord are all situated in layer V of the cortex (Kuypers, 1981; Wiesendanger, 1981; Jones, 1984). It was shown that the corticospinal pathway in the rabbit reaches spinal motor centers of the forelimbs (Haarsten and Verhaart, 1967; Armand, 1982, 1984).

Descending corticofugal neurons of layer VI were also identified by their antidromic response to stimulation of the ventrolateral thalamus. Differentiation of CF5 and CF6 neurons was determined on the basis of (1) a deeper position in the cortex of CF6 neurons as compared with CF5 neurons, (2) the presence of a "supernormal" period of reduced antidromic latency after a previous action potential in CF6s and its absence in CF5s, and (3) lower conduction velocities in CF6s as compared with CF5s. The CF6 neurons can be considered to be corticothalamic, because anatomical studies show that descending efferent axons of cortical layer VI terminate exclusively in the thalamus (Jones, 1984).

Corticocortical efferent neurons projecting to the contralateral hemisphere were identified by their antidromic response to stimulation of the contralateral motor or primary somatosensory cortex.

Corticocortical efferent neurons projecting to the ipsilateral hemisphere were identified by their antidromic response to stimulation of the ipsilateral primary somatosensory cortex. 

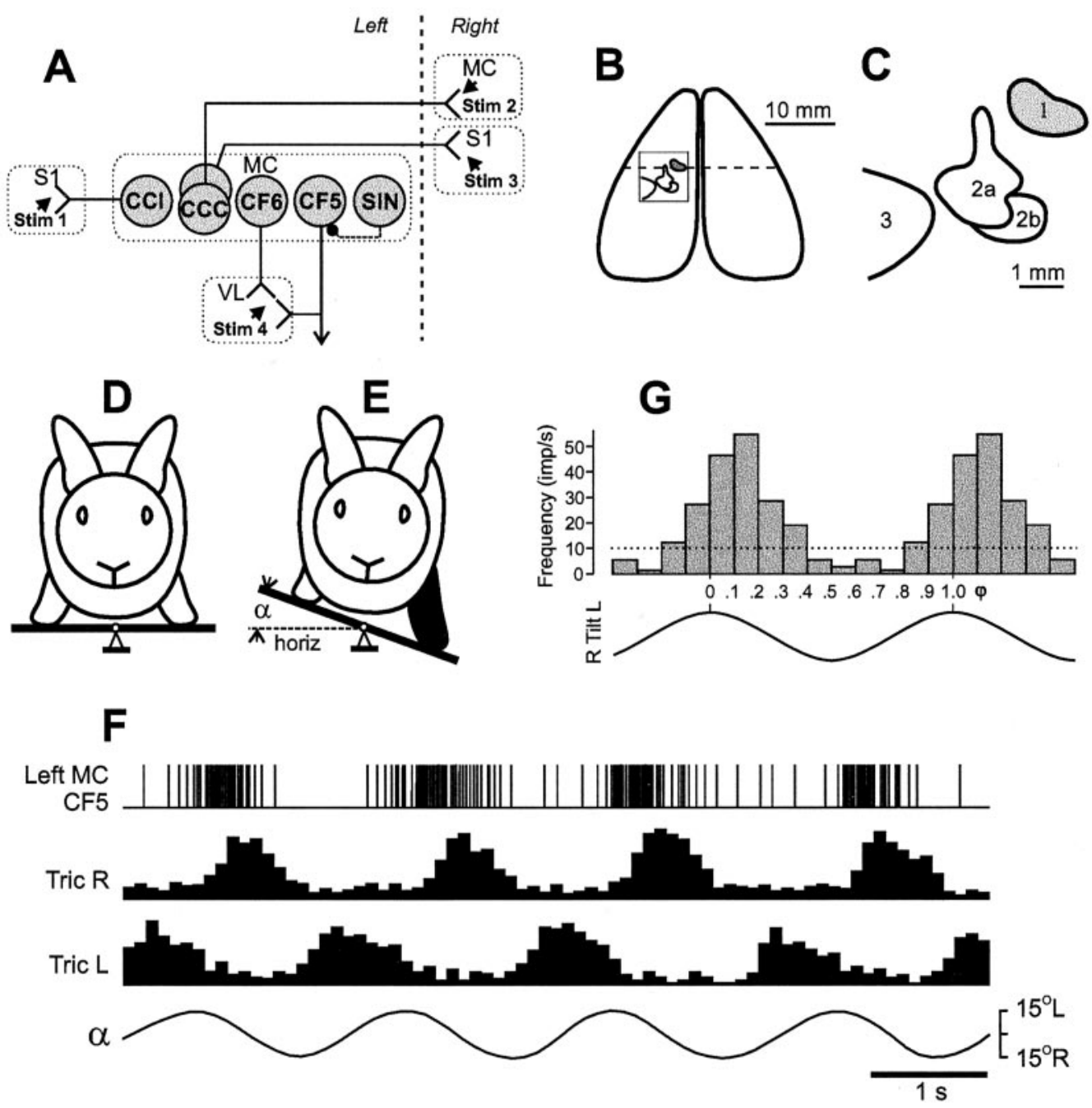

Figure 1. Experimental arrangement. $A$, Types of neurons that were recorded in the forelimb representation of the left motor cortex (MC). CCl, Corticocortical neurons projecting to the ipsilateral primary somatosensory cortex (S1); $C(C$, corticocortical neurons projecting to the contralateral motor (MC) or primary somatosensory cortex (S1); $(F 6$, corticofugal neurons of layer VI projecting to the ventrolateral thalamus (VL); $\mathrm{CF}$, corticofugal neurons of layer $\mathrm{V}$ with collaterals projecting to the ventrolateral thalamus. These neuron types were identified by their antidromic responses to electrical stimulation of the corresponding structures (Stim 1-Stim 4). SIN, Putative inhibitory interneurons were identified by their high-frequency orthodromic responses to stimulation of ventrolateral thalamus or a cortical site (Stim 1-Stim 4). B, C, Location of the motor limb representation in the rabbit (a typical example). B, Schematic drawing of the hemispheres (dorsal view) and the limb representations. The dashed horizontal line indicates the zero anteroposterior coordinate. The square area shown in $B$ is represented in $C$ with a higher magnification to show the relative positions of motor representation of the forelimb (1), the primary somatosensory representations of the forelimb (2a) and the hind limb (2b), as well as that of the whiskers (3). D, E, A rabbit with implanted electrodes was positioned on a platform that was tilted periodically in the frontal (roll) plane (amplitude, $\pm 15^{\circ}$ ). The rabbit tended to maintain the dorsal side-up orientation on the platform by extending the limbs on the side moving down. The forelimb with high extensor activity is shown in black in E. F, Periodic tilts of the platform caused alternating EMG responses of the left and right $m$. triceps and periodic responses of a CF5 neuron from the left motor cortex. The EMGs are presented as time histograms (Deliagina et al. 2000a); the spikes of the neuron were transformed into standard pulses for subsequent data processing. G, A frequency histogram for the CF5 neuron shown in $D$, averaged over eight tilt cycles. The cycle phase $(\varphi)$ is indicated; the peak of the left tilt was taken as the cycle onset. The activity of the neuron exceeding the threshold (interrupted line) was considered as a burst.

Putative inhibitory interneurons were identified as the neurons that did not respond antidromically to stimulation of any site tested but responded synaptically to stimulation of at least one site, i.e., the ventrolateral thalamus, sensory, or motor cortex, with a burst of spikes $(n \geq 3)$ at a peak frequency $>600 \mathrm{~Hz}$ (Swadlow, 1988, 1994).

The nonidentified neurons (NIs) in this study were the cells that did not respond antidromically to stimulation of any site (ventrolateral thalamus, ipsilateral and contralateral cortex) and also did not meet criteria for SINs (high-frequency response to stimulation). NI neurons could respond synaptically to some or all stimulated sites. The NI group was probably heterogeneous and included neurons projecting to the sites that were not stimulated, as well as interneurons.

Receptive field classification. The somatic receptive fields of each neuron were examined in the resting animals under conditions of head restraint. Stimulation was produced by palpation of muscle bellies, tendons, etc. and by passive movements of joints. The size of superficial receptive fields was determined by listening to the audio monitor and measuring the entire area from which action potentials could be elicited. A directional selectivity was assessed by comparing the number of spikes elicited by flexing the joint in the optimal direction with the direction opposite from optimal.

Postural tests. After a neuron was identified, the head of the animal was freed, and the animal was transferred to a tilting platform for postural tests. An arrangement for the tests is shown schematically in Figure 1, $D$ and $E$ [see also Deliagina et al. (2000a)]. The platform supporting the rabbit was tilted periodically in the frontal (roll) plane. Two types of tilt trajectories were used. All neurons were tested using a sine-like trajectory, with a period of $2-3 \mathrm{sec}$ and amplitude of $\pm 15^{\circ}$ (Fig. $1 F$ ). In addition, a part of the neurons was tested using a "trapezoid" trajectory with the transition between $-15^{\circ}$ and $+15^{\circ}$ positions lasting for $0.7-1 \mathrm{sec}$, and each position was maintained for $\sim 3 \sec$ (see Fig. $6 A$ ). The tilt angle (Fig. $1 E, F, \alpha)$ was measured with a potentiometric transducer. 
Rabbits were not trained to perform this postural task; however, they were easily engaged in it. During recordings, animals were always fully awake and tended to maintain the dorsal side-up orientation of the head and trunk so that the deviation of their sagittal planes from the vertical were much less than the platform tilt angle. In the previous study (Deliagina et al. 2000a) it was found that postural corrections are accomplished by extension of the limbs on the side moving down and flexion of the limbs on the opposite side (Fig. 1D,E). It was also found that these corrective limb movements are reflected clearly in the EMG of m. triceps (elbow extensor): the limb extension is associated with an increase of EMG, and the limb flexion is associated with a decrease of EMG. In the present study, therefore, the activation of $m$. triceps during the ipsilateral tilt (Figs. $1 F, 6 A$ ) was used to monitor the postural corrective responses.

Locomotion tests. After postural tests, the activity of several neurons was also tested during unrestrained locomotion complicated by the task of overstepping a series of barriers (five barriers $4.5 \mathrm{~cm}$ high and $2 \mathrm{~cm}$ thick placed $43 \mathrm{~cm}$ apart). Positive reinforcement (food) was used to engage rabbits in locomotion behavior. A detailed description on the arrangement for locomotion tests has been provided previously (Beloozerova et al., 2003). During locomotion, the duration of swing and stance phases of step of the right forelimb was recorded by measuring the electrical resistance between the foot and the ground.

Data processing. To analyze the tilt-related activity of a neuron, 5-10 successive tilt cycles were selected in which the m. triceps EMGs were most regular, that is, were positioned in approximately the same phase of the cycle and had similar amplitude (Fig. $1 F$ ).

In tests with sinusoidal tilts, each of the cycles was divided into 10 equal bins, and a histogram of spike activity was generated (the peak of the left tilt was taken as the cycle onset). Then the activity was averaged over the successive tilt cycles (Fig. $1 G$ ). The portion of the cycle during which the activity level exceeded $25 \%$ of the difference between the maximal and minimal frequencies in the histogram (Fig. $1 G$, dotted line) was defined as a burst, and the remaining portion was defined as an interburst period. In the neurons with a "deep" frequency modulation, that is, the neurons that were silent in one part of the cycle, all spikes were ascribed to the burst. For the burst and interburst periods, average frequencies were calculated. The coefficient of frequency modulation was defined as $M=$ $1-F_{\text {inter }} / F_{\text {burst }}$, where $F_{\text {burst }}$ and $F_{\text {inter }}$ are average frequencies during the burst and interburst periods, respectively.

For each neuron class, bursts of all individual neurons were plotted against the phase of the tilt cycle to show their phase distribution. The peak of the left tilt was taken as the cycle onset (Fig. $1 G$; see Figs. $3 A, 4 A$, $5 A$ ). A dependence of the cell group activity on the phase of the tilt cycle was characterized by (1) a histogram of the number of simultaneously active neurons and (2) a histogram of the mean frequencies (see Fig. $3 B, C)$.

The EMG activity was usually presented in the form of temporal histograms, with an "event" defined as a waveform that exceeded a threshold put slightly above the noise level (Deliagina et al. 2000a) (Fig. 1 F). In some experiments, the EMG signals were rectified and smoothed by filters with a time constant of $100 \mathrm{msec}$ (see Fig. 6A). In all experiments, the EMGs were modulated periodically in relation to a tilt cycle. With sinusoidal tilts, an increased level of activity occurred during ipsilateral tilting, typically between phases 0.1 and 0.6 .

In tests with trapezoid tilts, each of the cycles was divided into four intervals (Fig. $6 B, 1-4$ ), and the discharge frequency of a neuron was measured separately for each interval and then averaged over the successive tilt cycles. The activity in intervals 1 and 3 (during platform movement) was termed the dynamic response; the activity in intervals 2 and 4 (when the platform position was maintained) was termed the static response. To characterize the pattern of response in each neuron, we compared the discharge frequency in the four intervals of the tilt cycle, that is, during tilting in the contralateral direction (interval 1), during sustained contralateral tilt (interval 2), during tilting in the ipsilateral direction (interval 3), and during sustained ipsilateral tilt (interval 4).

In locomotion tests, the onset of the swing phase was taken as the beginning of the step cycle. The duration of each step cycle was divided into 10 equal bins. The discharge frequency in a bin was derived according to the method of Udo et al. (1982). The frequency histograms were smoothed using a moving filter with a span of three. For each neuron, the mean frequency of discharge, the coefficient of modulation $(M)$, and the preferred step phase were calculated (Beloozerova et al., 2003).

Statistical procedures. All quantitative data in this study are presented as the mean \pm SEM. The $t$ test was used to characterize statistical significance when comparing different means; significance level was set at $p=$ 0.05 .

Histological procedures. At the termination of the experiment, rabbits were deeply anesthetized with pentobarbital sodium and perfused with isotonic saline followed by a $10 \%$ Formalin solution. Frozen sections of $50 \mu \mathrm{m}$ thickness were cut in the regions of recording and stimulating electrodes. The tissue was stained for Nissl substance with cresyl violet. The motor and sensory cortex were identified by inspection of the microscopic characteristics of the tissue: the expression of granular layer IV and the relative sizes of pyramidal cells in layer V. Locations of stimulation electrodes in the corresponding structures were verified by observation of electrode track gliosis.

\section{Results}

\section{Responses of neurons to sinusoidal tilts}

Data were collected from a total of 18 tracks through the forelimb area in the left motor cortex of three rabbits. The activity of 90 identified neurons of different classes and 76 nonidentified neurons was recorded. During testing, rabbits were always fully awake and actively stabilized their dorsal side-up orientation.

\section{Corticofugal neurons of layer $\mathrm{V}$}

The mean discharge frequency of CF5 neurons during tilts was $11.3 \pm 1.3$ impulses $(\mathrm{imp}) / \mathrm{sec}$ (mean $\pm \mathrm{SEM})$. A response to tilts (a tilt-related modulation of the discharge frequency) was observed in the overwhelming majority of CF5s (24 of 27, or 90\%) (Fig. 2A). The modulation was usually very pronounced, as is illustrated for one of the neurons in Figure $1 F$. Typically, the modulation pattern was similar in all successive tilt cycles and in repeated tests. The frequency in the burst averaged over all modulated neurons $(16.3 \pm 1.5 \mathrm{imp} / \mathrm{sec})$ was much higher $(p<$ $0.0001)$ than in between the bursts $(2.7 \pm 0.5 \mathrm{imp} / \mathrm{sec})($ Fig. $2 B)$, so that the coefficient of frequency modulation was as large as $83.9 \pm 3.2 \%$ (Fig. 2C).

Figure $3 A$ shows a distribution of the phases of activity of 24 modulated CF5 neurons over the tilt cycle, with a mid-burst position indicated. Most neurons fired one burst per cycle. Phases of activity of different neurons were distributed almost evenly over the cycle; however, in a histogram of the number of simultaneously active neurons (Fig. $3 B$ ), one can see a slight increase of this value in the first half of the cycle (that is, during tilting toward the contralateral side, phases $0-0.5$ ) as compared with the second half of the cycle (tilting toward the ipsilateral side, phases $0.5-$ $1.0)$. In these two parts of the cycle, the mean percentage of active neurons was, respectively, $60.0 \pm 2.1$ vs $50.0 \pm 3.5 \%(p=0.02)$. Similarly, the mean frequency in phases $0-0.5(11.6 \pm 0.4 \mathrm{imp} /$ $\mathrm{sec})$ was slightly higher $(p=0.03)$ than the mean frequency in phases $0.5-1.0(10.7 \pm 0.7 \mathrm{imp} / \mathrm{sec})($ Fig. $3 C)$.

\section{Putative inhibitory interneurons}

The mean discharge frequency of SINs during tilts was $35.4 \pm 4.2$ $\mathrm{imp} / \mathrm{sec}$, which is much higher than that of CF5 neurons $(11.3 \pm$ $1.3 \mathrm{imp} / \mathrm{sec} ; p=0.02)$. The response to tilt was observed in all 28 SINs (Fig. $2 A$ ). The frequency in the burst averaged over all neurons $(44.5 \pm 5.1 \mathrm{imp} / \mathrm{sec})$ was much higher $(p=0.002)$ than in between the bursts $(21.6 \pm 3.3 \mathrm{imp} / \mathrm{sec})$ (Fig. $2 B)$, so that the $M$ was as large as $56.0 \pm 3.0 \%$ (Fig. $2 C$ ). Both the mean frequency in the burst and in between the bursts were much higher than those in CF5 neurons ( $p=0.0001$ and $p=0.0002$, respectively) (Fig. 
A

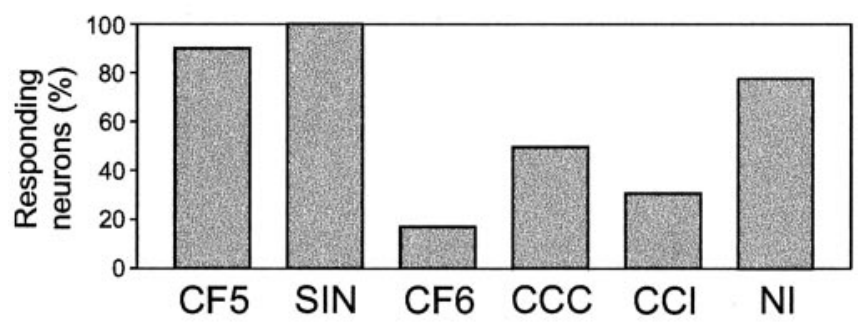

B
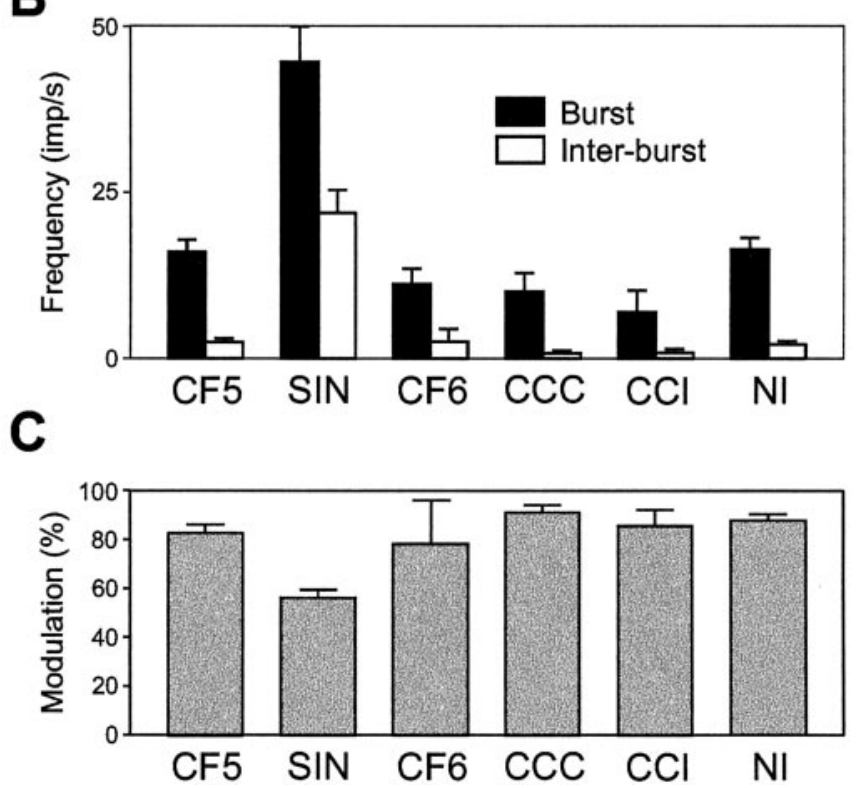

Figure 2. Population characteristics of different classes of neurons. $A$, Proportion of neurons responding to tilts in relation of their total number. $B$, Frequency in the burst and between the bursts. C, Coefficient of frequency modulation $M=1-F_{\text {inter }} / F_{\text {burst }}$ (Mean \pm SEM are shown.)

$2 B)$; however, the $M$ in SINs was smaller than in CF5 neurons $(56.0 \pm 3.0$ vs $83.9 \pm 3.2 \% ; p=0.0001)$ (Fig. $2 C$ ).

Figure $4 A$ shows a distribution of the phases of activity of 28 SINs over the tilt cycle, with a mid-burst position indicated. Most neurons fired one burst per cycle. Phases of activity of different neurons were distributed almost evenly over the cycle. This was reflected in the histogram of the number of simultaneously active neurons (Fig. $4 B$ ) as well as in the histogram of mean frequencies (Fig. $4 C$ ). The mean percentage of active neurons in phases $0-0.5$ $(60.7 \pm 5.4 \%)$ did not differ significantly from that in phases $0.5-1.0(56.0 \pm 2.7 \%)$. Similarly, the mean frequency in phases $0-0.5(35.0 \pm 1.4 \mathrm{imp} / \mathrm{sec})$ did not differ from that in phases $0.5-1.0(34.5 \pm 1.0 \mathrm{imp} / \mathrm{sec})$.

In one animal we classified SINs into two groups depending on where, in the track, a neuron was encountered: superficial to CF5 neurons $(n=6)$ or among CF5s and deeper $(n=6)$. It was found that the SINs recorded in superficial layers were less active than those recorded in deeper layers. The mean frequency in the burst for the superficial SINs was $15.5 \pm 3.1 \mathrm{imp} / \mathrm{sec}$ versus $51.0 \pm 8.1 \mathrm{imp} / \mathrm{sec}$ for the deep ones $(p=0.001)$.

\section{Corticofugal neurons of layer VI}

A response to tilt was observed in a very small proportion of CF6 neurons (2 of 12, or 17\%) (Fig. 2A). Although not statistically significant, the burst frequency of responding neurons had a tendency to be lower than that of CF5s $(11.3 \pm 0.6$ vs $16.3 \pm 1.8$
A CF5s from left MC, $\mathrm{n}=24$

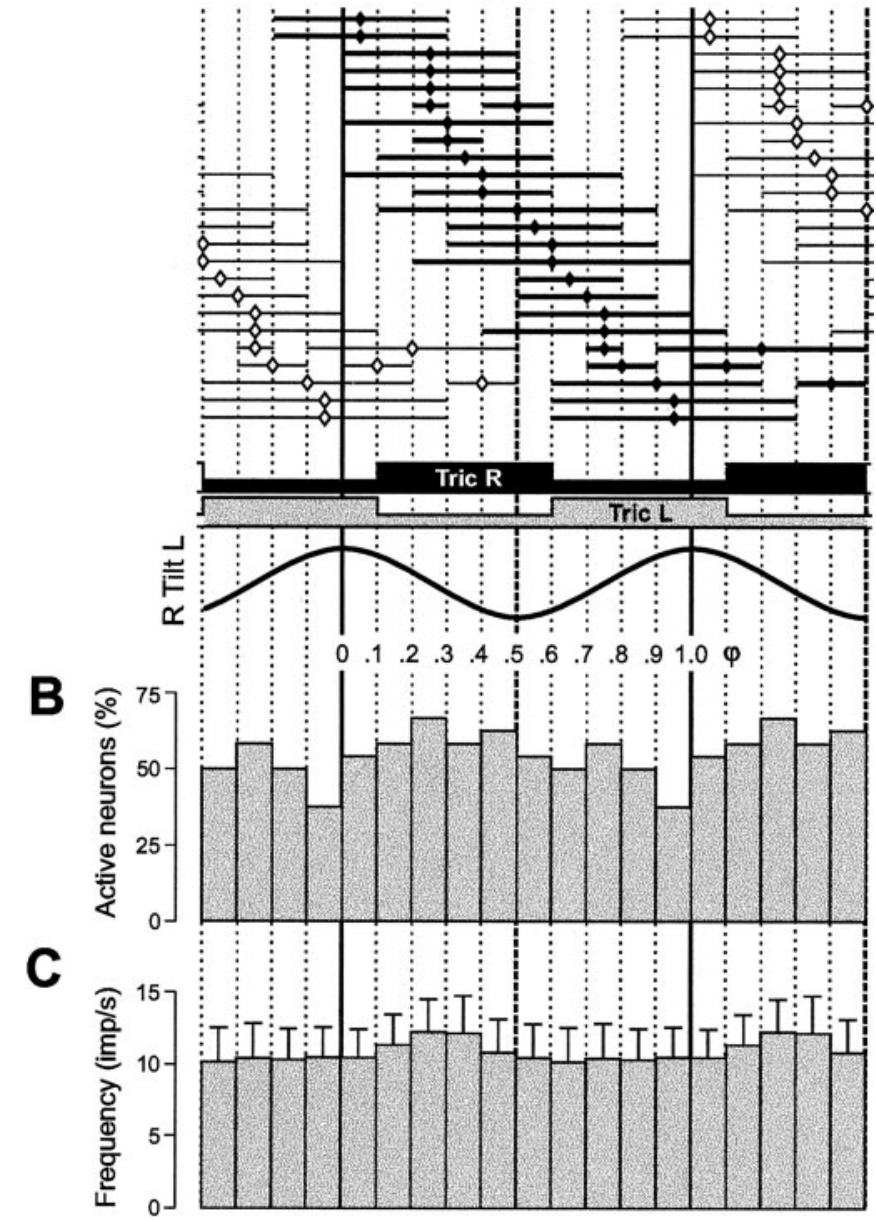

Figure 3. Phase characteristics of $\mathrm{CF} 5$ neurons. A, Phase distribution of bursts of individual neurons in the tilt cycle, with the mid-burst phase indicated. The activity of each neuron is presented in bold only one time in the cycle. The neurons are rank ordered according to the mid-burst phase of the first burst. $B, A$ histogram of the relative number of active neurons in different phases of the tilt cycle. $C, A$ histogram of the mean frequency of active neurons in different phases of the tilt cycle. In $A$, the periods of increased and decreased activity of the right and left m. triceps (Tric R and Tric L) are shown schematically. In this and subsequent figures, $\varphi$ is the cycle phase, and the peak left tilt is taken as the cycle onset.

$\mathrm{imp} / \mathrm{sec}$ ) (Fig. 2 B). One of these neurons had a mid-burst phase in the first half of the tilt cycle, and one had a mid-burst phase in the second half.

\section{Corticocortical neurons with contralateral projections}

The response to tilt was observed in one-half of CCC neurons ( 5 of 10) (Fig. 2A). Although not statistically significant, the burst frequency of responding neurons had a tendency to be lower than that of CF5s $(10.0 \pm 3.0 \mathrm{vs} 16.3 \pm 1.8 \mathrm{imp} / \mathrm{sec})$ (Fig. $2 \mathrm{~B})$. Three of these neurons had a mid-burst phase in the first half of the tilt cycle, and two had a mid-burst phase in the second half.

\section{Corticocortical neurons with ipsilateral projections}

The response to tilt was observed in approximately one-third of CCI neurons (4 of 13) (Fig. 2A). The burst frequency of responding neurons was lower than that of CF5s $(7.1 \pm 3.1$ vs $16.3 \pm 1.8$ $\mathrm{imp} / \mathrm{sec} ; p=0.03$ ) (Fig. $2 B$ ). Three of these neurons had a midburst phase in the first half of the tilt cycle, and one had a midburst phase in the second half. 
A SINs from left MC, $n=28$
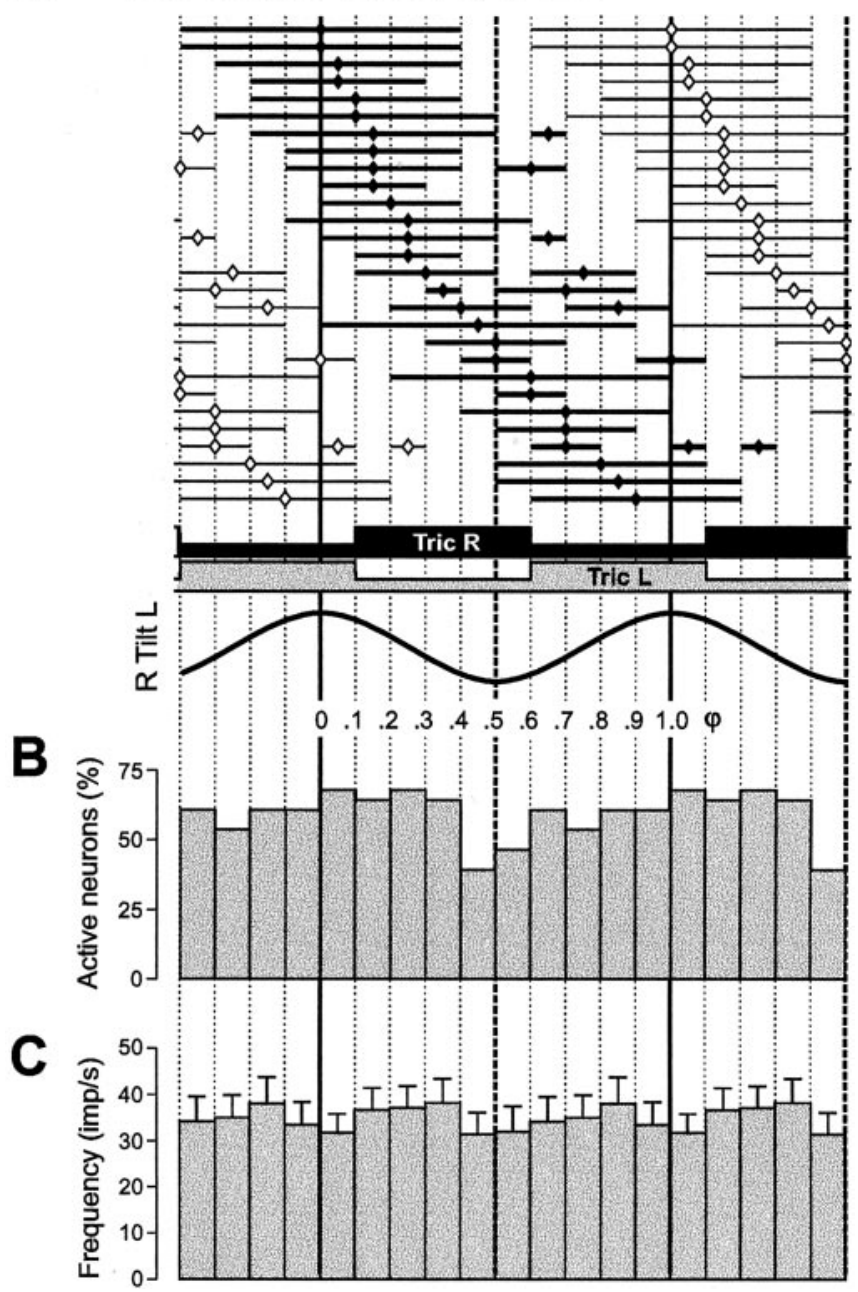

Figure 4. Phase characteristics of SINs. A, Phase distribution of bursts of individual neurons in the tilt cycle, with the mid-burst phase indicated. $B, A$ histogram of the relative number of active neurons in different phases of the tilt cycle. $C$, A histogram of the mean frequency of active neurons in different phases of the tilt cycle. Designations are as in Fig. 3.

\section{Nonidentified neurons}

All studied characteristics of the activity of NI neurons were very similar to those of CF5 neurons. The response to tilt was observed in the majority of NI neurons (59 of 76, or 78\%) (Fig. 2A). The tilt-related modulation of the discharge frequency in NIs was usually very pronounced: the mean frequency in the burst $(16.5 \pm 1.5 \mathrm{imp} / \mathrm{sec})$ was much higher $(p=0.0001)$ than in between the bursts $(2.2 \pm 0.4 \mathrm{imp} / \mathrm{sec})$, so that the coefficient of frequency modulation was as large as $87.9 \pm 1.9 \%$ (Fig. $2 B, C$ ).

Figure $5 A$ shows a distribution of the phases of activity of 59 modulated NI neurons over the tilt cycle. Most neurons fired one burst per cycle. The phase distribution was not even, which was seen clearly in the histogram of the number of active neurons (Fig. $5 B$ ) and in the histogram of mean frequencies (Fig. 5C). The mean percentage of active neurons in phases $0-0.5$ (53.2 \pm $3.4 \%)$ was larger $(p=0.001)$ than that in phases $0.5-1.0(35.6 \pm$ $2.1 \%)$. Similarly, the mean frequency in phases $0-0.5(9.0 \pm 0.2$ $\mathrm{imp} / \mathrm{sec})$ was higher $(p=0.004)$ than the mean frequency in phases $0.5-1.0(7.7 \pm 0.3 \mathrm{imp} / \mathrm{sec})$. This pattern of cycle-related modulation of NI neurons was similar to that of CF5 neurons (Fig. $3 B, C$, compare Fig. $5 B, C$ ).
A Nis from left MC, $\mathrm{n}=59$

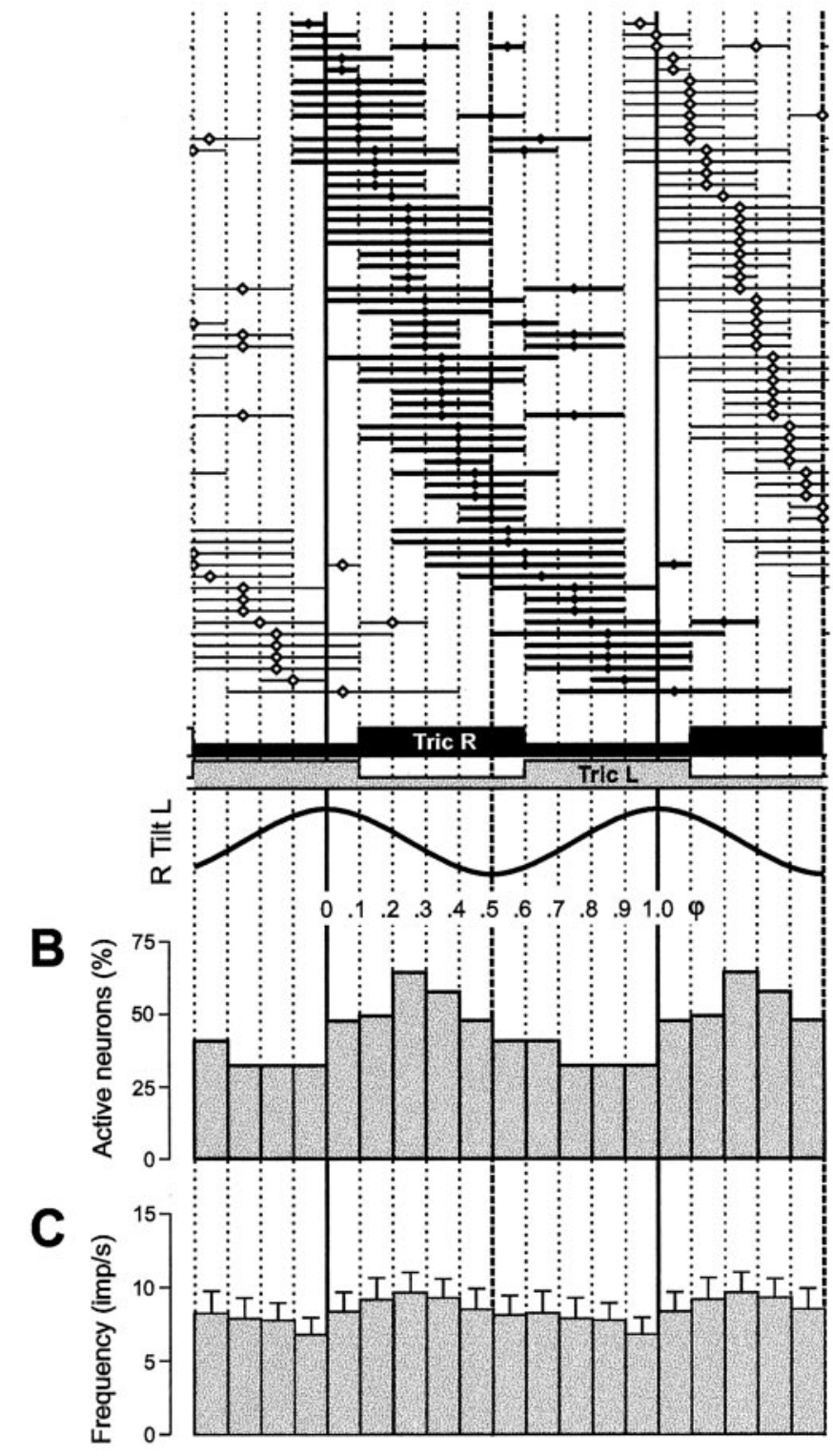

Figure 5. Phase characteristics of NI neurons. $A$, Phase distribution of bursts of individual neurons in the tilt cycle, with the mid-burst phase indicated. $B, A$ histogram of the relative number of active neurons in different phases of the tilt cycle. $C, A$ histogram of the mean frequency of active neurons in different phases of the tilt cycle. Designations are as in Fig. 3.

\section{Responses of cortical neurons to trapezoid tilts}

Tests with sinusoidal tilts clearly demonstrated the involvement of some groups of cortical neurons in the tilt-related activity; however, these tests did not allow us to distinguish between the static and dynamic components of the activity of the neurons, that is, between their position-related and movement-related responses. To reveal these two components, we used trapezoid tilts. A test with trapezoid tilts (Fig. 6A,B) was performed in 16 CF5 neurons, 15 SINs, and 23 NI neurons. All tested neurons responded to trapezoid tilts.

In each neuron class (CF5, SIN, and NI), all neurons could be divided into two groups (Fig. $6 C, \mathrm{co}, \mathrm{i}$ ) that were preferentially active with the contralateral tilt (Fig. $6 B$, intervals 1 and 2 ) or with the ipsilateral tilt (Fig. 6B, intervals 3 and 4). In the CF5 and NI classes, the co and i groups were of approximately equal size, 
A

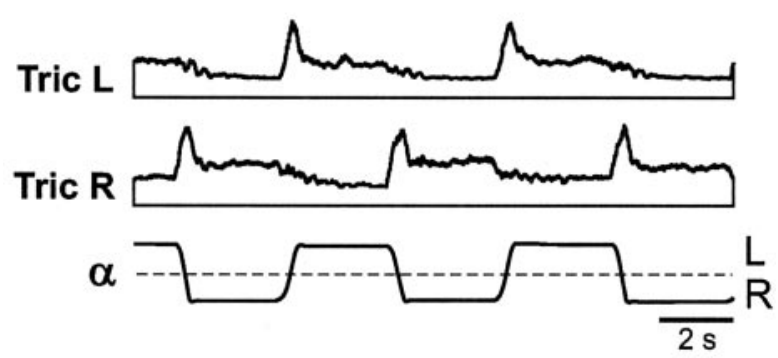

B Neuron

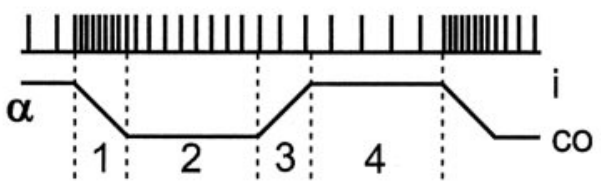

C

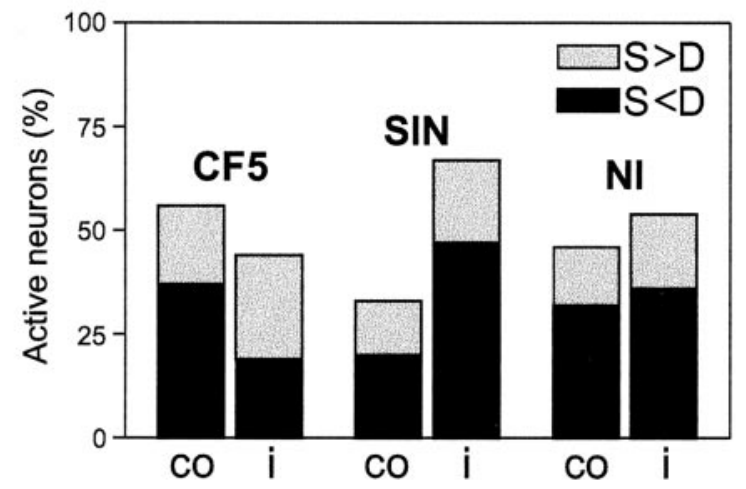

D

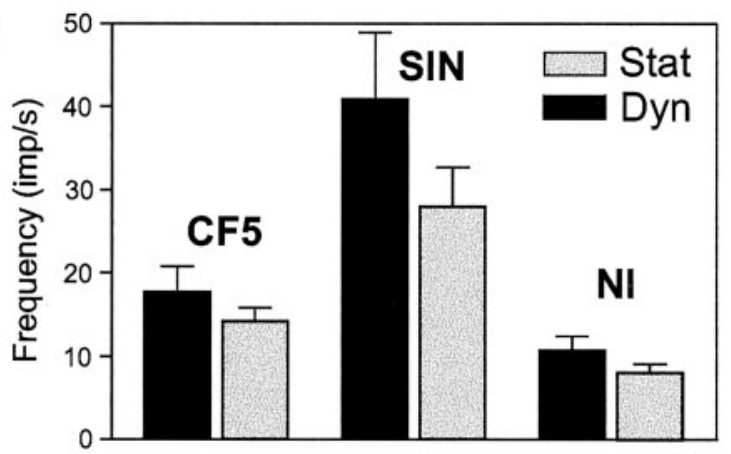

Figure 6. Responses of different classes of cortical neurons to trapezoid tilts. A, Activity of the left and right $\mathrm{m}$. triceps during tilts. The EMG signals were rectified and smoothed. (Note dynamic and static components in the EMGs.) B, Schematic representation of the tilt cycle and of a response of a neuron (co and $\mathrm{i}=$ contralateral and ipsilateral tilt, respectively). The discharge frequency was calculated separately for each of the intervals (1-4) and then averaged over the successive tilt cycles. C, Relative number of neurons in each class (CF5, SIN, NI) responding preferentially to contralateral tilt (co group) and ipsilateral tilt (i group). For each group, a proportion of neurons with a dynamic response prevailing over a static response $(D>S)$ and that with a static response prevailing over a dynamic response $(S>D)$ are indicated. $D$, The discharge frequency in the dynamic and static responses evoked by tilt in a preferred direction, in different classes of neurons.

whereas in the SIN class, the i group was two times larger than the co group.

Each group of neurons could be subdivided further into two subgroups, depending on which response was stronger: the dy- namic one (activity during tilting) or the static one (activity in a tilted position). In all groups (except for the CF5 i group), the "dynamic" subgroup was approximately two times larger than the "static" one. In the CF5 i group, the static subgroup was slightly larger than the dynamic one (Fig. 6C).

Figure $6 D$ shows, for each class of neurons, the mean frequency of the discharge evoked by the tilt in a "preferable" direction, for both its dynamic and static components. One can see that the dynamic responses slightly exceeded the static responses. The value of dynamic responses to trapezoid tilts was similar to the value of responses to sinusoidal tilts (Fig. $2 B$, Burst). One can thus conclude that the activity of cortical neurons reflects both the value of the maintained tilt angle and the change of this angle.

Responses of cortical neurons to somatosensory stimulation We tried to associate postural responses in profoundly modulated neuron groups (CF5, SIN, and NI) with their responses to somatosensory stimulation in quiescent animals. In total, 56 neurons were tested ( 16 CF5s, 17 SINs, and $23 \mathrm{NIs}$ ) for somatosensory receptive fields. Of these, no peripheral receptive fields were found in eight neurons (14\%). Nevertheless, these neurons were profoundly modulated in postural tests. Two neurons were inhibited by somatosensory stimulation; one of them responded to tilts.

Forty-six neurons $(82 \%)$ had excitatory receptive fields in the right forelimb. Most of these fields were "deep": the cells responded to palpation of muscles or movements in joints, or both, but not to stimulation of fur alone. Receptive fields of 5 neurons (9\%) were confined to the wrist region (including 2 with receptive fields on the footpad), those of 12 neurons (21\%) were on the elbow, and those of 17 neurons (30\%) included shoulder muscles and joint. Receptive fields of 10 neurons (18\%) covered all of the forelimb from wrist to shoulder. In 25 responding neurons $(45 \%)$, the responses had a directional preference: 9 neurons (16\%) preferred flexion to extension, 11 neurons $(20 \%)$ preferred extension to flexion, 2 neurons ( $7 \%$ ) were activated during abduction, and 3 neurons (11\%) were activated during adduction of the right forelimb.

We have not found any simple correlation between responses of the neurons to somatosensory stimulation and their postural responses. Of four neurons responding to passive elbow or wrist flexion, or both, three neurons were active in the first half of the tilt cycle (i.e., during extension of the limb), and one neuron was active in the second half (i.e., during limb flexion). All nine neurons that were activated by large passive forward-backward movements in the shoulder joint responded well to tilts, despite the fact that postural corrections evoked by tilts did not include such forward-backward movements. Similarly, all five neurons that were activated by large passive abduction-adduction movements of the limb were well modulated in postural tests, despite the absence of such movements. One of the two cells that had a receptive field on the footpad was preferentially active during the ipsilateral tilt, and the other one was preferentially active during the contralateral tilt. Thus, one can conclude that the somatosensory signals, which are received by cortical neurons in a quiescent animal, cannot be responsible for modulation of their activity during active stabilization of posture. One can suggest that somatosensory information is processed in the spinal postural networks before it reaches the cortex (Deliagina and Orlovsky, 2002). "Efference copy" signals can also play a role in the modulation of descending postural commands, as was demonstrated previously for the descending locomotor commands (Orlovsky et al., 1999). 


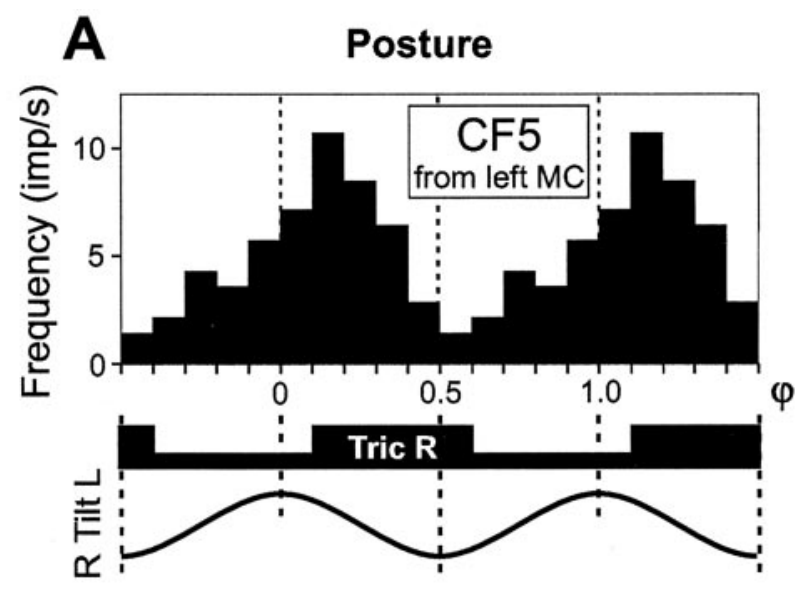

B Locomotion

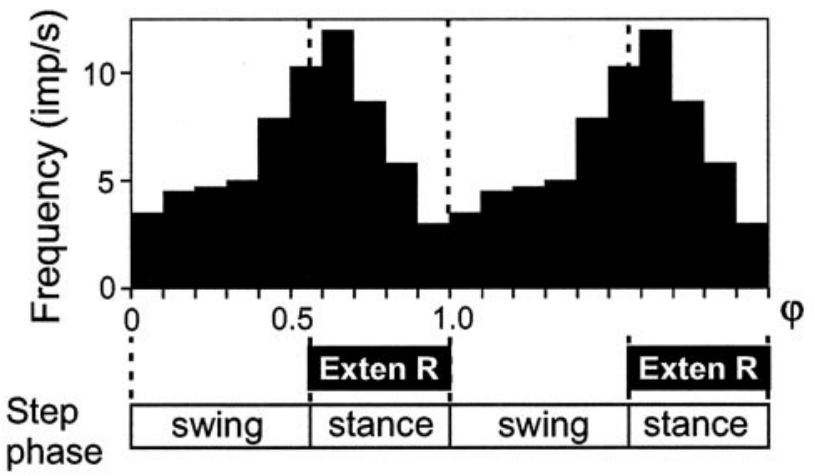

C

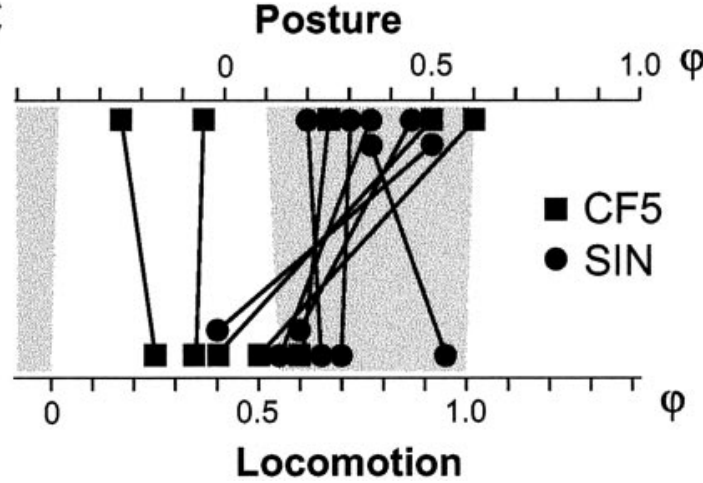

Figure 7. Comparison of activity of cortical neurons in postural and locomotor tasks. $A, B, A$ histogram of activity of the $C F 5$ neuron recorded initially in the postural task $(A)$ and then in the locomotor task $(B)$. In $B$, the swing and stance phases of the step cycle of the contralateral (right) forelimb are indicated, as well as an approximate phase of the extensor activity. C, Position of the mid-burst point in the tilt cycle and in the step cycle for 11 neurons recorded in the two motor tasks. The phase of increased extensor activity is indicated for both cycles (shaded area).

\section{Comparison of activity of cortical neurons in postural and locomotor tasks}

Figure 7, $A$ and $B$, shows the activity of a CF5 neuron recorded during a postural task and a complex locomotion task, when the animal was overstepping a series of barriers. In the postural task (Fig. 7A), the neuron was mostly active during contralateral tilting, and the peak of its activity was timed to the initial part of the EMG burst in the contralateral m. triceps. During locomotion (Fig. $7 B$ ), this neuron had a peak of activity in the stance phase of the step cycle, that is, when the extensor muscles of the contralat- eral forelimb are active. Thus, in both tasks, the relationships between the phases of activity of the neuron and the extensor activity were similar.

Altogether, five CF5 neurons and six SINs were recorded in both motor tasks. All of the neurons were rhythmically modulated in both tasks. The mean discharge frequency and the depth of modulation either were similar in the two tasks (Fig. $7 A, B$ ) or were larger in the postural task; however, the sample size was too small to make any conclusions on the statistical significance of the differences between the tasks.

For each neuron, the mid-burst position was defined in both tilt and step cycles. These positions are shown in Figure $7 C$, where the tilt and step cycles are presented so that the mid-point of the period of increased extensor activity in the tilt cycle is aligned with the mid-point of the extensor (stance) phase of the step cycle. One can see that in three neurons a transition from the postural to the locomotor task was associated with a considerable change of the mid-burst position, whereas in eight neurons the change was relatively small; in both motor tasks the mid-burst point was situated in the analogous (extensor or nonextensor) parts of the cycle. Comparison of population activities in the two tasks will be provided in Discussion.

\section{Discussion}

Posture-related activity in the motor cortex

The main result of this study is that in the fully awake animals standing on a platform, the activity of many neurons in the motor cortex is strongly correlated with the tilts of the platform and with the corrective postural responses caused by these tilts. Among the task-related neurons were all of the SINs, most of the CF5s, which are known to project to subcortical motor nuclei and the spinal cord (Kuypers, 1981; Wiesendanger, 1981; Jones, 1984), and most of the NIs tested. The coefficient of frequency modulation was high in all of these populations. These findings strongly suggest that the motor cortex participates in the feedback mode of postural control and that cortical mechanisms do supplement the basic, brainstem-cerebello-spinal postural mechanisms.

To estimate a degree of participation of the motor cortex in postural control, one can compare the response characteristics of cortical neurons in the postural task with those in other motor tasks. The three rabbits used in this study were also used in the concurrent experiments that examined the activity of motor cortical neurons during two locomotor tasks, i.e., walking on a flat terrain ("simple locomotion") and walking along a path with a series of barriers ("complex locomotion") (Beloozerova et al., 2003). Overstepping the barriers is a very complicated motor behavior that requires visuomotor coordination and participation of the motor cortex (Beloozerova and Sirota 1988, 1993; Drew 1988, 1993). A striking similarity was found when comparing the activity of cortical neurons during the postural task and during the complex locomotion task. In the both tasks, the overwhelming majority of CF5, SIN, and NI neurons exhibited a clear modulation, with approximately similar characteristics. The mean frequency for CF5s was $11.3 \pm 1.3 \mathrm{imp} / \mathrm{sec}$ in the postural task versus $8.8 \pm 1.3 \mathrm{imp} / \mathrm{sec}$ in the locomotor task. For SINs these values were $35.4 \pm 4.2$ versus $34.1 \pm 3.2 \mathrm{imp} / \mathrm{sec}$; in NI neurons, they were $8.1 \pm 0.9$ versus $8.8 \pm 1.2 \mathrm{imp} / \mathrm{sec}$. The coefficient of frequency modulation for CF5s was $83.9 \pm 3.2 \%$ in the postural task versus $79 \pm 2 \%$ in the complex locomotor task. For SINs these values were $56.0 \pm 3.0$ versus $64.2 \pm 3.3 \%$; in NI neurons, they were $88.0 \pm 2.0$ versus $67.2 \pm 2.2 \%$. Similar results were obtained for the few neurons that were tested during both the postural and locomotor tasks. One can thus conclude that the 
cortical "output" neurons (CF5s), the subpopulation of interneurons, and the whole population of not identified neurons are all periodically modulated in the postural task to a degree not less than they are in the complex locomotion task for which the cortical participation was shown to be essential (Trendelenberg, 1911; Liddell and Phillips, 1944; Beloozerova and Sirota, 1993).

In contrast to CF5s, SINs, and NI neurons, the proportion of corticofugal neurons of layer VI and of corticocortical neurons with ipsilateral and contralateral projections that were active during postural corrections was relatively small, and their discharge frequencies were low. When tested in the concurrent study (Beloozerova et al., 2003) during locomotion, the CCI, CCC, and CF6 neurons also demonstrated extremely low activity levels and a poor association of that activity with both the simple and complex locomotion tasks.

On the grounds of the low activity and the weak modulation of CCI, CCC, and CF6 neurons during both posture maintenance and locomotion, one can suggest that these neuronal populations do not have any significant role in the control of these behaviors. The inactivity of many callosal neurons suggests that coordination between the two symmetrical limbs in both postural and locomotor tasks does not require interaction of the two hemispheres, and is achieved at the brainstem-spinal level. The low level of activity in CF6 neurons during both postural corrections and locomotion is most surprising. These neurons are usually considered to be an essential part of the thalamocortical loop (Sherman and Guillery, 2001). Our finding suggests that, at least during postural corrections and locomotion, functioning of this loop is based mostly on the feedback signals that are transmitted to the thalamus by CF5 neurons.

\section{Correlation between cortical output and postural corrections}

Maintenance of the dorsal side-up posture on the tilting platform is controlled primarily because of a redistribution of activity between the extensor muscles of the left and right limbs (Figs. $1 F$, $6 A$ ). An increase of extensor activity on the side moving down leads to limb extension (Fig. $1 E$ ), and a decrease of extensor activity on the side moving up leads to limb flexion under the effect of body weight [see also Deliagina et al. (2000a)]. The activity of elbow flexor ( $m$. biceps) during tilts was rather low at all times and was not modulated (T. G. Deliagina, unpublished observation). The overall muscular activity pattern in each limb, therefore, can be roughly approximated as alternating periods of high and low levels of activity in the antigravity (extensor) muscles (Deliagina et al. 2000a, their Fig. 6A).

How does cortical output, that is, the activity of descending CF5 neurons from the forelimb representation in the motor cortex, correlate with muscular activity in the corresponding (contralateral) forelimb? From Figure 3 one can see that considerable changes of extensor activity during tilt cycle were accompanied by only slight changes in cortical output as characterized by the number of active CF5 neurons and their frequency. The subpopulation of CF5 neurons firing preferentially during the period of higher extensor activity only slightly exceeded in number and frequency the subpopulation firing preferentially during the period of lower extensor activity.

The simplest hypothesis about the functional role of the two subpopulations (presumably projecting to the subcortical and spinal motor centers) is that the "extensor phase" neurons participate in the activation of extensors, whereas the neurons of the opposite phase contribute to the inhibition of extensors. An inhibitory action on extensors, exerted by some corticospinal neurons, was shown in a number of studies; this inhibition has often been associated with excitation of flexors (Agnew et al., 1963; Alstermark et al., 1984; Alstermark and Sasaki 1985; Armstrong and Drew 1985; Kassler and Cheney 1985). The low level of flexor activity when the "dual role" subpopulation of CF5 neurons is active in the postural task can be explained by suggesting that cortical commands are not sufficient to activate flexors without support from the basic brainstem-cerebello-spinal mechanism.

An additional possible role of CF5s is that, via their projections to the ventrolateral thalamus, these neurons modulate the transmission of signals that reach the motor cortex via this route.

\section{Origin of tilt-related cortical activity}

The postural control system is driven by sensory signals of three modalities, somatosensory, vestibular, and visual, that supplement each other (Horak and Macpherson 1995). A relative contribution of these inputs to the postural task used in the present study has been investigated previously (Deliagina et al. 2000a). It was demonstrated that the animals were capable of maintaining the dorsal side-up trunk orientation on the tilting platform after elimination of vestibular and visual inputs, whereas vestibular input was important for head stabilization. It was argued that corrections of the trunk posture are generated by the spinal and brain stem postural mechanisms driven by somatosensory input originating from the load receptors in the limbs. It therefore seems likely that the tilt-related cortical activity, revealed in the present study, is also driven by somatosensory input activated by tilts of the platform, although this question must be addressed directly.

In the present study it was found that, for each class of neurons, the bursts of individual neurons are distributed over the tilt cycle (Figs. 3-5). Individual neurons also strongly differed in their activity under static versus dynamic conditions (Fig. 6). This diversity of the response patterns suggests that different neurons are driven by different components of the somatosensory input, most likely after they have been processed in the spinal postural networks. The functional significance of such diversity of the response patterns is not clear.

The tilt-related signals from the spinal cord and brainstem can reach the motor cortex and affect its output (CF5) neurons via different routes. One of these is the input via the ventrolateral thalamus. A part of it that is mediated by SINs (Strick and Sterling, 1974; White, 1989; Swadlow, 2002) was investigated in the present study. The activity of SINs was rhythmically modulated during postural corrections; therefore, one can hypothesize that SINs contributed to shaping of the cortical output. The role of other inputs, including direct excitatory input from the ventrolateral thalamus, in modulation of CF5 neurons remains unclear.

In conclusion, the present study has demonstrated that the activity of some classes of neurons in the motor cortex is closely related to a highly automatic motor activity, the maintenance of the body posture.

\section{References}

Adkins RJ, Cegnar MR, Rafuse DD (1971) Differential effects of lesions of the anterior and posterior sygmoid gyri in cats. Brain Res 30:411-414.

Agnew RF, Preston JB, Whitlock DG (1963) Patterns of motor cortex effects on ankle flexor and extensor motoneurons in the "pyramidal" cat preparation. Exp Neurol 8:247-263.

Alstermark B, Sasaki S (1985) Integration in descending motor pathways controlling the forelimb in the cat. 13. Cortical effects in shoulder, elbow, wrist and digit motoneurons. Exp Brain Res 59:353-364.

Alstermark B, Lundberg A, Sasaki S (1984) Integration in descending motor pathways controlling the forelimb in the cat. 10. Inhibitory pathways to forelimb motoneurons via C3-C4 propriospinal neurons. Exp Brain Res $56: 279-292$. 
Armand J (1982) The origin, course and termination of corticospinal fibers in various mammals. Prog Brain Res 57:329-360.

Armand J (1984) The pyramidal tract. Recent anatomic and physiologic findings. Rev Neurol (Paris) 140:309-329.

Armstrong DM, Drew T (1985) Forelimb electromyographic responses to motor cortex stimulation during locomotion in the cat. J Physiol (Lond) 367:327-351.

Bard P, Macht MB (1958) The behavior of chronically decerebrated cat. In: Neurological basis of behaviour (Wolstenholme GEW, O'Connor CM, eds), pp 55-71. London: Churchill.

Beloozerova IN, Sirota MG (1988) Role of motor cortex in control of locomotion. In: Stance and motion. Facts and concepts (Gurfinkel VS, Ioffe ME, Massion J, Roll JP, eds), pp 163-176. New York: Plenum.

Beloozerova IN, Sirota MG (1993) The role of the motor cortex in the control of accuracy of locomotor movements in the cat. J Physiol (Lond) 461:1-25.

Beloozerova IN, Sirota MG, Swadlow HA (2003) Activity of different classes of neurons of the motor cortex during locomotion. J Neurosci 23:1087-1097.

Bishop PO, Burke W, Davis R (1962) The identification of single units in central visual pathways. J Physiol (Lond) 162:409-431.

Chambers WW, Lin CN (1957) Corticospinal tract of the cat. An attempt to correlate the pattern of degeneration with deficits in reflex activity following neocortical lesions. J Comp Neurol 108:23-56.

Deliagina TG, Orlovsky GN (2002) Comparative neurobiology of postural control. Curr Opin Neurobiol 12:652-657.

Deliagina TG, Arshavsky YI, Orlovsky GN (1998) Control of spatial orientation in a mollusc. Nature 393:172-175.

Deliagina TG, Orlovsky GN, Selverston A, Arshavsky YI (1999) Neuronal mechanisms for the control of body orientation in Clione. 1. Spatial zones of activity of different neuron groups. J Neurophysiol 82:687-699.

Deliagina TG, Beloozerova IN, Popova LB, Sirota MG, Swadlow H, Grant G, Orlovsky GN (2000a) Role of different sensory inputs for maintenance of body posture in sitting rat and rabbit. Motor Control 4:439-452.

Deliagina TG, Beloozerova IN, Sirota MG, Swadlow H, Orlovsky GN (2000b) Activity of different classes of neurons in the rabbit motor cortex related to postural corrections. Soc Neurosci Abstr 26:1484.

Deliagina TG, Zelenin PV, Fagerstedt P, Grillner S, Orlovsky GN (2000c) Activity of reticulospinal neurons during locomotion in the freely behaving lamprey. J Neurophysiol 83:853-863.

Drew T (1988) Motor cortical cell discharge during voluntary gait modification. Brain Res 457:181-187.

Drew T (1993) Motor cortical activity during voluntary gait modifications in the cat. I. Cells related to the forelimbs. J Neurophysiol 70:179-199.

Dubrovsky B, Garcia-Rill E, Surkes MA (1974) Effects of discrete precruciate cortex lesions on motor behavior. Brain Res 82:328-333.

Fuller JH, Schlag J (1976) Determination of antidromic excitation by the collision test: problems of interpretation. Brain Res 122:283-298.

Gould HJ (1986) Body surface maps in the somatosensory cortex of rabbit. J Comp Neurol 243:207-233.

Haarsten AB, Verhaart WJC (1967) Cortical projections to brain stem and spinal cord by way of the pyramidal tract and the bundle of Bagley. J Comp Neurol 129:189-202.

Hensler K (1988) The pars intercerebralis neurone PI(2)5 of locusts: convergent processing of inputs reporting head movements and deviations from straight flight. J Exp Biol 140:511-533.

Horak F, Macpherson J (1995) Postural orientation and equilibrium. In: Integration of motor, circulatory, respiratory and metabolic control during exercise. Handbook of Physiology, Section 12 (Shepard J, Rowell L, eds), pp 1-39. New York: Oxford UP.
Jones EG (1984) Laminar distribution of cortical efferent cells. In: Cerebral cortex. Cellular components of the cerebral cortex (Peters A, Jones EG, eds), Vol 1. New York: Plenum.

Kassler RG, Cheney PD (1985) Characteristics of cortico-motoneuronal postspike facilitation and reciprocal suppression of EMG activity in the monkey. J Neurophysiol 53:959-978.

Kuypers HGJM (1981) Anatomy of the descending pathways. In: Handbook of physiology. Sect 1, The nervous system. Vol II, Motor control, Part I (Brookhart JM, Mountcastle VB, Brooks VB, Geiger SR, eds), pp 597-666. Baltimore: Williams \& Wilkins.

Liddell EGT, Phillips CG (1944) Pyramidal section in the cat. Brain 67:1-9.

Macpherson JM, Deliagina TG, Orlovsky GN (1997) Control of body orientation and equilibrium in vertebrates. In: Neurons, networks, and motor behavior (Stein PSG, Grillner S, Selverston AI, Stuart DG, eds), pp 257-267. Cambridge, MA: MIT.

Magnus R (1924) Körperstellung. Berlin: Springer.

Massion J (1998) Postural control systems in developmental perspective. Neurosci Biobehav Rev 22:465-472.

Mori S (1987) Integration of posture and locomotion in acute decerebrate cats and in awake, freely moving cats. Prog Neurobiol 28:161-195.

Orlovsky GN, Deliagina TG, Grillner S (1999) Neuronal control of locomotion. From mollusc to man. Oxford: Oxford UP.

Sherman SM, Guillery RW (2001) Exploring the thalamus. New York: Academic.

Strick PL, Sterling P (1974) Synaptic termination of afferents from the ventrolateral nucleus of the thalamus in the cat motor cortex. A light and electron microscopy study. J Comp Neurol 153:77-106.

Swadlow HA (1988) Efferent neurons and suspected interneurons in binocular visual cortex of the awake rabbit: receptive fields and binocular properties. J Neurophysiol 59:1162-1187.

Swadlow HA (1989) Efferent neurons and suspected interneurons in S-1 vibrissa cortex of the awake rabbit: receptive fields and axonal properties. J Neurophysiol 62:288-308.

Swadlow HA (1990) Efferent neurons and suspected interneurons in S-1 forelimb representation of the awake rabbit: receptive fields and axonal properties. J Neurophysiol 63:1477-1498.

Swadlow HA (1994) Efferent neurons and suspected interneurons in motor cortex of the awake rabbit: axonal properties, sensory receptive fields and subthreshold synaptic inputs. J Neurophysiol 71:437-453.

Swadlow HA (1998) Neocortical efferent neurons with very slowly conducting axons: strategies for reliable antidromic identification. J Neurosci Methods 79:131-141.

Swadlow HA (2002) Thalamocortical control of feed-forward inhibition in awake somatosensory "barrel” cortex. Philos Trans R Soc Lond B Biol Sci 357:1717-1727.

Trendelenberg W (1911) Untersuchungen uber reizlose vorubergehende Aussaltung am Zentralnervensystem. III. Die extermitaten Region der Grosshirninde. Pflügers Arch 137:515-544.

Udo M, Kamei H, Matsukawa K, Tanaka K (1982) Interlimb coordination in cat locomotion investigated with perturbation. II. Correlates in neuronal activity of Deiter's cells of decerebrate walking cats. Exp Brain Res 46:438-447.

White EL (1989) Cortical circuits. Boston: Birkhauser.

Wiesendanger M (1981) The pyramidal tract. Its structure and functional considerations. In: Handbook of behavioral neurobiology, Vol 5 (Lowe AL, Luschei AF, eds), pp 401-491. New York: Plenum.

Zelenin PV, Deliagina TG, Grillner S, Orlovsky GN (2000) Postural control in the lamprey: a study with a neuro-mechanical model. J Neurophysiol $84: 2880-2887$ 